\title{
Development of Pipe Holding Mechanism for Pipe Inspection Robot Using Flexible Pneumatic Cylinder
}

\author{
Kyujun Choi, Shujiro Dohta, Tetsuya Akagi ${ }^{\mathrm{a}}$ and Shota Ninomiya \\ Department of Intelligent Mechanical Engineering, Okayama University of Science \\ 1-1, Ridai-cho, Kita-ku, Okayama, 700-0005, Japan
}

\begin{abstract}
A pipe inspection robot is useful to reduce the inspection cost. In the previous study, a novel pipe inspection robot using a flexible pneumatic cylinder that can move forward along to the pipe by changing the robot's body naturally was proposed and tested. In this paper, to improve its mobility for a corner of a pipe, the thin pipe holding mechanism using pneumatic bellows was proposed and tested. As a result of its driving test, the holding performance of the mechanism was confirmed.
\end{abstract}

\section{Introduction}

In Japan, it is found that the corresponding to $5 \%$ of the total length of water supply pipe had been already past the statutory useful life of 40 years [1]. Water pipe lines are very complex and includes quite a lot of corners and joints. The cost of the inspection of pipe will be reduced by using inspection robot [2-4]. In the case of pipe inspection, the robot is required higher mobility. In ideal, it is more desirable that the shape of the robot changes naturally because of the lower energy consumption and decreasing the time for traveling. In our pervious study, a pipe inspection robot that consists of a flexible sliding mechanism using the flexible pneumatic cylinder that can travel in the narrower space smoothly by changing the body according to the shape of the pipe had been proposed and tested [5-7]. In the next step, it is necessary to improve its mobility for corners or joints. In this paper, the thin pipe holding mechanism using pneumatic bellows is proposed and tested so as not to prevent the smooth movement in a complex bending pipe. The holding performance of the tested mechanism is also investigated.

\section{Pipe inspection robot using flexible pneumatic cylinder}

Figure 1 shows the double ball type flexible pneumatic cylinder developed in our previous study [8]. As shown in Fig. 1(a), the cylinder consists of a flexible tube as a cylinder, two steel balls ( $\Phi 9 \mathrm{~mm})$ as a cylinder head, and a slide stage. The slide stage has two brass rollers $(\Phi 4 \mathrm{~mm})$ set on the inner bore of the stage to press and deform the tube. The tube between two steel balls is pinched by the slide stage. When the pressure is applied to one side of the cylinder, the slide stage moves forward and backward. The slide stage can move even if the cylinder bends. The flexible cylinder can be used by two ways as shown in Figs.1(b) and (c). Figure 1(b) shows an ordinary rod-less type cylinder: the

\footnotetext{
${ }^{a}$ Corresponding author : akagi@are.ous.ac.jp
} 
slide stage moves on the curved tube while the cylinder tube is fixed. On the other hand, the cylinder tube can move through the slide stage when the slide stage is fixed as shown in Fig.1(c). This usage can be easily realized because the cylinder tube is more light-weight compared with a usual metallic cylinder. In this study, both operations are used properly in a sliding mechanism.

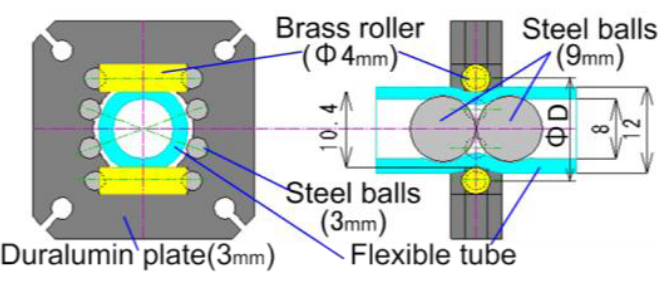

(a) Construction of the cylinder.

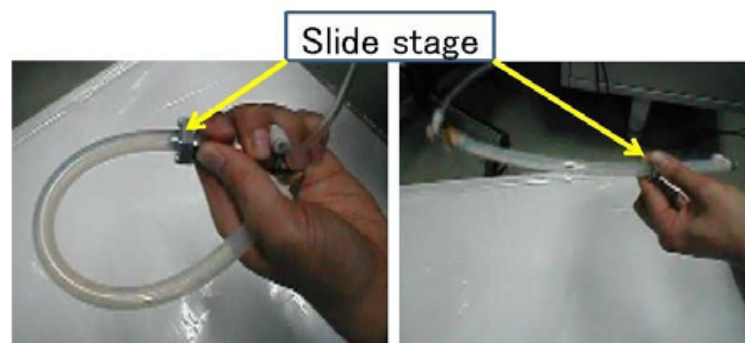

(b) Cylinder tube is fixed.

(c) Slide stage is fixed.

Figure 1. Construction and operation of flexible pneumatic cylinder.

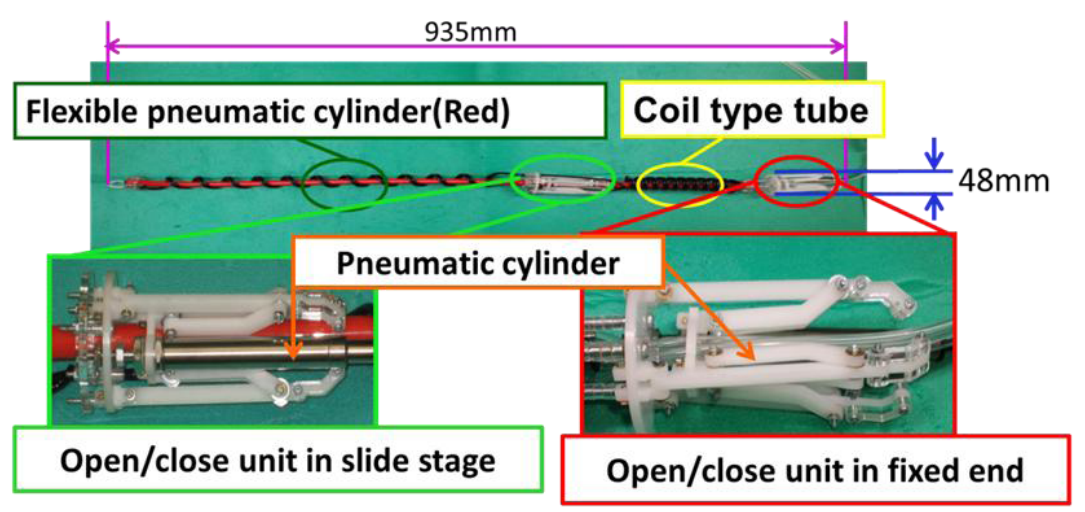

Figure 2. Flexible sliding mechanism for inspection robot.

Figure 2 shows a flexible sliding mechanism using a flexible pneumatic cylinder and two open/close units developed in the previous study [6]. The coil type of pneumatic supply tube is used as a supply pipe to the open/close unit on the slide stage and to the top end chamber of the cylinder. The open/close unit can expand the claw toward radial direction using the typical pneumatic cylinder. The flexible pneumatic cylinder is located into the coil tube. By this method, the smart configuration of air supply pipes from the end of the mechanism can be realized. Figure 3 (a) shows the operating principle of the sliding mechanism. The operating principle is as follows. First, the open/close unit at the end of the mechanism opens so that the claws can hold the pipe (2). Next, the right side chambers of the flexible cylinder are pressurized, then the slide stage with the open/close unit can slide toward the top end of the cylinder (3). When the slider reaches at the end of the cylinder, the open/close unit opens to hold the pipe (4). Then, the open/close unit at the end of mechanism closes (5), and the left side chambers of the cylinders are pressurized. At the same time, the flexible cylinder with end open/close unit can move forward (6). By repeating these operations from (2) to (6), the mechanism can move forward as an inchworm. Figure 3 (b) shows the view of movement of the tested inspection robot using the sliding mechanism when the robot passes through the pipe with the inner diameter of $100 \mathrm{~mm}$. The size of the robot is $1.1 \mathrm{~m}$ in length and $48 \mathrm{~mm}$ in outer diameter. The whole mass of the robot including the pneumatic driving system is $0.78 \mathrm{~kg}$. The motion for passing through pipe is as follows. First, from the far front of pipe tee, the bending unit of the robot was bent toward the desired direction (2). While keeping the bending motion, the robot moved forward. Then, the acrylic cover 
was inserted into the desired pipe. The robot can move forward along to the pipe by changing the robot's body naturally. Figure 3(b) (3) shows the case when the robot passes through the pipe elbow.

In the same manner as the case of the pipe tee, by bending toward the desired direction in the far front of the corner, the robot can easily move at the corner. The moving speed passing through both corners is almost same speed of moving straight (about $140 \mathrm{~mm} / \mathrm{s}$ ). However, it was also observed that the open/close unit was often caught on the corner while bending. Therefore, a thinner pipe holding mechanism will be required.

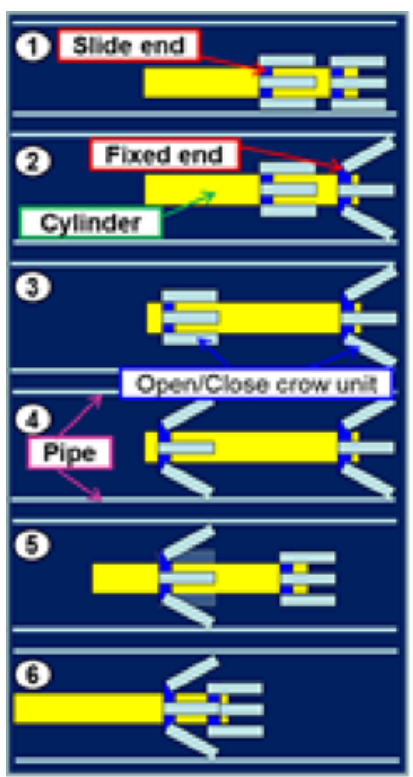

(a) Operating principle.
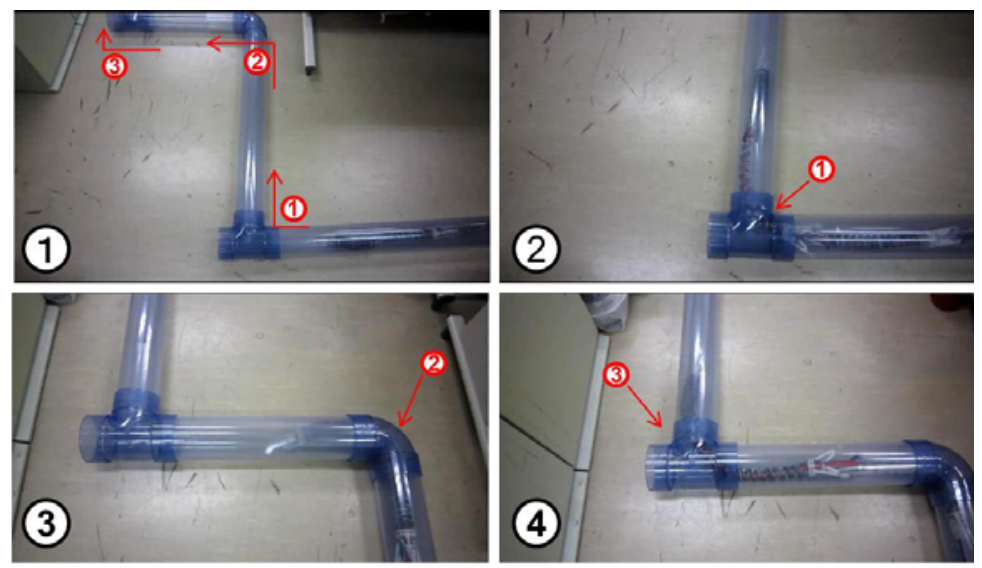

(b) View of movement of inspection robot in complex pipe.

Figure 3. Operating principle of sliding mechanism and movement of inspection robot in complex pipe.

\section{Thinner pipe holding mechanism}


Figure 4(a) shows the view of the thinner pipe holding mechanism proposed in this study. The mechanism consists of three acrylic claws that can expand toward radial direction and three bellows. Each claw is located every 120 degs. from the center of the mechanism. Each bellow is also set between the claw and the slide stage. The size of the mechanism is $72 \mathrm{~mm}$ in outer diameter and 31 $\mathrm{mm}$ in length. The mass of the mechanism is very small, that is $75 \mathrm{~g}$.

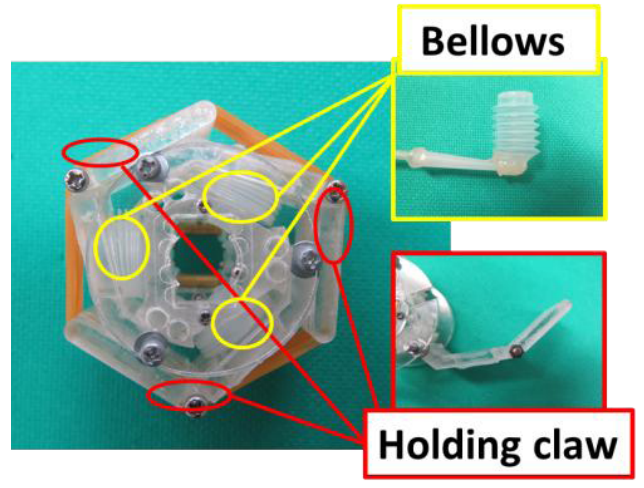

(a) View of the mechanism.

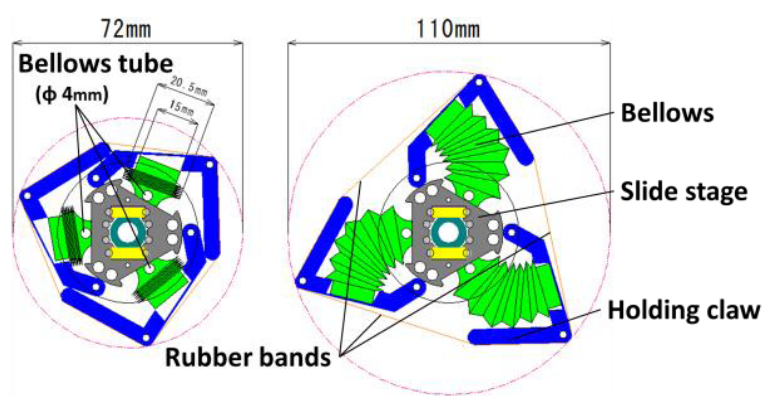

(b) Operating principle of the mechanism.

Figure 4. Proposed thinner pipe holding mechanism.

Figure 4(b) shows the operating principle of the holding mechanism. The operating principle is as follows. When the supply pressure is applied to three bellows, the bellows expands towards the radial direction. The maximum outer diameter of $110 \mathrm{~mm}$ can be obtained when the supply pressure of 200 $\mathrm{kPa}$ is applied. When the supply pressure is released, the mechanism returns to its original shape by restoring force of rubber bands that cover the claws. By using this device, the mechanism can hold the pipe surely. Figure 5 shows the view of operation of the tested pipe holding mechanism. As a result of pipe holding test, the mechanism in the pipe with inner diameter of $100 \mathrm{~mm}$ can hold a load of $19.6 \mathrm{~N}$. It is considered that this holding ability is sufficient to be applied for the pipe inspection robot. The travelling test of the proposed inspection robot in a pipe with corners is our future work.
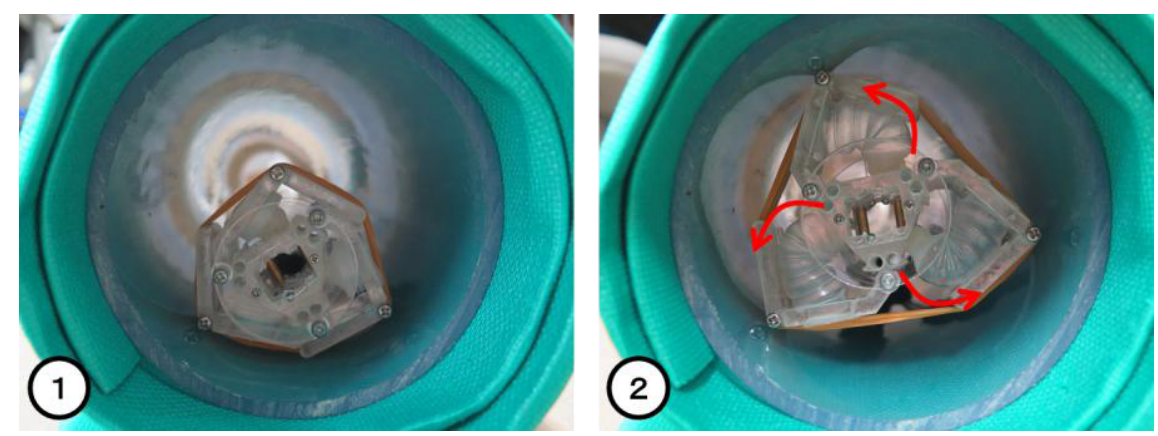

Figure 5. Operation of the tested pipe holding mechanism in a pipe.

\section{Conclusions}

In order to improve the mobility of the pipe inspection robot using flexible pneumatic cylinder when passing through corners, the thinner pipe holding mechanism using pneumatic bellows was proposed and tested. The holding performance of the mechanism was also investigated. As a result, the mechanism can hold a load of $19.6 \mathrm{~N}$. It can be confirmed that the mechanism is useful for the pipe inspection robot because of its thinner body and sufficient holding ability. 


\section{Acknowledgements}

This research was supported by the Ministry of Education, Culture, Sports, Science and Technology of Japan through a Financial Assistance Program of QOL Innovative Research (2012-2016).

\section{References}

1. http://ja.wikipedia.org/wiki/\%E6\%B0\%B4\%E9\%81\%93\%E7\%AE\%A1.

2. J. Yuan et.al., Development of an inspection robot for long distance transmission pipeline on-site overhaul, Int. J. Industrial Robot, Vol. 36, pp.546-550 (2009)

3. E. Dertien et.al., Development of an inspection robot for small diameter gas distribution mains, Proc. 2011 IEEE Int. Conf. R\&A, pp.281-294 (2011)

4. K. Nagatani et.al., Development of a Transformable Mobile Robot with a Variable Wheel Diameter, J. Robotics and Mechatronics, Vol.19, No.3, pp.252-257 (2007)

5. T. Kawasaki et.al., Development of a Flexible Sliding Mechanism Using Flexible Pneumatic Cylinder for Rescue Robot, Proc. FLUCOME2011, 41.pdf, pp.1-6 (2011)

6. T. Akagi et.al., Development of sliding type inspection robot using flexible pneumatic cylinder, Int. J. Advanced Mechatronic Systems, Vol.5, pp. 87-94 (2013)

7. T. Akagi et.al., Pipe Inspection Robot Using Flexible Pneumatic Cylinder Built-in Pneumatic Driving System, Proc. Int. Forum on Systems and Mechatronics, pp. 1-6 (2014)

8. T. Akagi et.al., Development of Wearable Pneumatic Actuator and Multiport Pressure Control Valve, J. Robotics and Mechatronics, Vol.17, No.5, pp. 529-536 (2005) 